\title{
The influence of generalist predators in spatially extended predator-prey systems
}

\section{Chakraborty, Subhendu}

Published in:

Ecological Complexity: An International Journal on Biocomplexity in the Environment and Theoretical Ecology

Link to article, DOI:

10.1016/j.ecocom.2015.06.003

Publication date:

2015

Document Version

Early version, also known as pre-print

Link back to DTU Orbit

Citation (APA):

Chakraborty, S. (2015). The influence of generalist predators in spatially extended predator-prey systems. Ecological Complexity: An International Journal on Biocomplexity in the Environment and Theoretical Ecology, 23, 50-60. https://doi.org/10.1016/j.ecocom.2015.06.003

\section{General rights}

Copyright and moral rights for the publications made accessible in the public portal are retained by the authors and/or other copyright owners and it is a condition of accessing publications that users recognise and abide by the legal requirements associated with these rights.

- Users may download and print one copy of any publication from the public portal for the purpose of private study or research.

- You may not further distribute the material or use it for any profit-making activity or commercial gain

- You may freely distribute the URL identifying the publication in the public portal 


\title{
The influence of generalist predators in spatially extended predator-prey systems
}

\author{
Subhendu Chakraborty ${ }^{\mathrm{a}, \mathrm{b}, *}$ \\ ${ }^{a}$ ICBM, Carl von Ossietzky University Oldenburg, Carl von Ossietzky Str. 9-11, 26111 Oldenburg, Germany \\ ${ }^{b}$ VKR Centre for Ocean Life, National Institute of Aquatic Resources, Technical University of Denmark, \\ Charlottenlund Slot, Jægersborg Allé, DK-2920 Charlottenlund, Denmark
}

\begin{abstract}
The presence of generalist predators is known to have important ecological impacts in several fields. They have wide applicability in the field of biological control. However, their role in the spatial distribution of predator and prey populations is still not clear. In this paper, the spatial dynamics of a predator-prey system is investigated by considering two different types of generalist predators. In one case, it is considered that the predator population has an additional food source and can survive in the absence of the prey population. In the other case, the predator population is involved in intraguild predation, i.e., the source of the additional food of the predator coincides with the food source of the prey population and thus both prey and predator populations compete for the same resource. The conditions for linear stability and Turing instability are analyzed for both the cases. In the presence of generalist predators, the system shows different pattern formations and spatiotemporal chaos which has important implications for ecosystem functioning not only in terms of their predictability, but also in influencing species persistence and ecosystem stability in response to abrupt environmental changes. This study establishes the importance of the consideration of spatial dynamics while determining optimal strategies for biological control through generalist predators.

Keywords: Generalist predator, additional food, intraguild predation, Turing instability, pattern formation, biological control
\end{abstract}

1. Introduction

Predator-prey interactions are determinants of the composition and distribution of species in a community. These interactions mainly depend on the type of predators and their activities. Generalist

\footnotetext{
${ }^{*}$ Corresponding author

Email addresses: subc@aqua.dtu.dk (Subhendu Chakraborty), Tel.: +45 3588 3347, Fax: +45 33963434 (Subhendu Chakraborty)
} 
predators, that utilize a possibly wide variety of food sources, play a crucial role in determining the dynamics of such communities. For example, raccoons (a medium-sized mammal native to North America) are an important part of our ecosystem as they feed on insects, small mammals and birds, eggs, and plant foods. For the last couple of decades, generalist predators have received considerable attention in the context of invasion ecology and pest control, which are important for sustainable and integrated pest-management strategies (Rosenheim et al., 1995; Symondson et al., 2002; Magal et al., 2008; Crowder and Snyder, 2010). Generalist predators affect pest populations in various ways. The ability of generalist predators to ingest new invasive pests can have drastic effects on the local pest populations. For example, the control of the local tomato pest Bemisia tabaci populations enhances by the generalist predator Macrolophus pygmaeus in the presence of invasive alien pest Tuta absoluta (Jaworski et al., 2013). However, predator-prey interactions generally occur over a wide range of spatial and temporal scales and the spatial components of ecological interactions play an important role in shaping ecological communities. In this respect, spatial patterns are ubiquitous in nature and often change the temporal dynamics of the system (Malchow et al., 2008; Seurout, 2009; Chakraborty et al., 2015). But, till now, very less attention has been paid to investigate the role of generalist predators under the influence of heterogeneous environments.

In the past, several researchers used mathematical models to investigate the role of generalist predators on ecological dynamics. Most of them modeled generalist predators simply by using a sigmoidal Holling type III response (which reflects prey switching at low prey concentrations) without considering another food source (Rosenzweig, 1971; Steele and Henderson, 1992; Hesaaraki and Moghadas, 2001; Xu et al., 2004; Kar and Matsuda, 2007; Morozov and Petrovskii, 2009; Chakraborty and Feudel, 2014). However, this is inconsistent with the fact that generalist predators can survive in the absence of focal prey. Only a few studies investigated the role of generalist predators in the presence of additional food source in predator-prey systems. Spencer and Collie (1995) and Chakraborty and Chattopadhyay (2008) considered a linear growth term to represent the growth of a predator due to the additional food source apart from the growth due to focal prey species. van Baalen et al. (2001) examined the switching between a focal prey and alternative food source by considering the alternative food density as constant. van Leeuwen et al. (2007) discussed the validation of different functional 
responses for generalist predators and found that generalist predators can have both stabilizing and destabilizing effects on the system dynamics. Similar to Spencer and Collie (1995), Magal et al. (2008) also considered additional food for a generalist predator, but Holling type II functional response for the uptake of focal prey rather than a sigmoidal functional response. Recently, Erbach et al. (2013) modeled a generalist predator by density-dependent birth rate of the predator and a linear death rate. Moreover, there are also few studies where generalist predators are modeled in the presence of spatial heterogeneity. Some of them did not consider an extra food source for the generalist predator (Rosenzwig, 1973; Segel and Levin, 1976) whereas others did not investigate different pattern formations due to the presence of generalist predators (Magal et al., 2008; Kumari, 2013). In the present paper, I investigate how a generalist predator affects the spatial distribution of the populations and results in different pattern formations.

Here, a two-dimensional reaction-diffusion predator-prey system is considered where the predator is a generalist predator and has additional food source apart from the focal prey population. The main focus of the paper is to investigate how the presence of a generalist predator affects the spatial distribution of the predator and prey populations. The dynamics with linear as well as density-dependent birth rate of the predator as considered in Spencer and Collie (1995) and Erbach et al. (2013), respectively, is investigated. Furthermore, the situation when the additional food source coincides with the food source of the focal prey is also examined. This kind of predation is known as intraguild predation (also mixotrophy), a special case of generalist predation (Gagnon et al., 2011; Kang and Wedekin, 2013). In this case, the predator is involved in competition for the common resources with the prey in addition to predate on them. For example, the scorpion Paruroctonus mesaenis eats smaller arachnid and insect predators together with the prey of these predators (Polis and McCormick, 1987). Several other examples of intraguild predation from natural communities can be found in Polis et al. (1989). The rest of the article is organized as follows: Section 2 deals with the model considering linear and density dependent birth rate of the predator due to the additional food source. Specifically, the model with linear birth rate of the predator due to the additional food and diffusion is presented in Section 2.1. Section 2.2 and 2.3 consist of the linear stability analysis of the model without diffusion and Turing instability conditions of the model with diffusion, respectively. In Section 2.4, different 
dynamics of the system are examined numerically and different types of pattern formation are shown in subsection 2.5 and 2.6. In subsection 2.7, a system with density-dependent birth rate of the predator is stated and the results are compared with the results from the previous section. A model with an intraguild predator is presented and analyzed in Section 3. Finally, the paper ends with a discussion.

\section{A predator-prey model with a generalist predator}

\subsection{Basic model structure}

Here, a reaction-diffusion system with a prey and a generalist predator in the presence of additional food for the predator is considered in the following form:

$$
\begin{aligned}
& \frac{\partial n}{\partial t}=r_{1} n\left(1-\frac{n}{K}\right)-\frac{g n p}{h+n}+D_{1}\left(\frac{\partial^{2} n}{\partial x^{2}}+\frac{\partial^{2} n}{\partial y^{2}}\right) \\
& \frac{\partial p}{\partial t}=r_{2} p+\frac{e g n p}{h+n}-m H(p) p+D_{2}\left(\frac{\partial^{2} p}{\partial x^{2}}+\frac{\partial^{2} p}{\partial y^{2}}\right)
\end{aligned}
$$

where $n(x, y, t)$ and $p(x, y, t)$ denote the densities of the prey and the predator, respectively, at location $(x, y) \in \Re^{2}$ and time $t \geq 0, r_{1}$ and $K$ are the intrinsic growth rate and carrying capacity of the prey population, respectively, $g$ is the prey capturing rate by the predator, $h$ is the corresponding handling time, $e$ is the efficiency of converting prey into predator biomass $(e<1), r_{2}$ is the growth rate of the predator due to the additional food source, $D_{1}$ and $D_{2}$ are diffusion coefficients of prey and predator, respectively, $m H(p)$ is the death rate of the predator. Concerning the form of $H(p)$, several functions are used in literature with various ecological interpretations (Steele and Henderson, 1992). However, in the present work, to take into account the predation of higher-order predators on the generalist predator that is not explicitly included in the model, a quadratic closure term is chosen, i.e., $H(p)=p$. This form of $H(p)$ assumes that the higher predator population changes in proportion with the generalist predator (Steele and Henderson, 1981).

Let, $\Omega$ be the two-dimensional bounded connected square domain with $\partial \Omega$ as boundary, and $\frac{\partial}{\partial \eta}$ be the outward drawn normal derivative on the boundary. In $\Omega$, the following initial conditions are taken for system 1

$n(0, x, y)=n_{0}(x, y)>0, p(0, x, y)=p_{0}(x, y)>0, \forall(x, y) \in \Omega$

and the zero-flux boundary conditions are chosen as 
It is to be noted here that the general model structure of system (1) is similar with the model of Magal et al. (2008) where a host-parasitoid model was considered to search for the conditions to restrict the growth of the host population. However, the motivation of the present work is completely different; here different pattern formations in a predator-prey system are investigated depending on the additional food source. In the following, the conditions for local asymptotic stability and Turing instability will be derived.

\subsection{Linear stability analysis}

To study Turing instability, first we need to analyze the stability criteria of the non-diffusive version of system (1). The corresponding non-diffusive model is

$$
\begin{aligned}
& \frac{d n}{d t}=r_{1} n\left(1-\frac{n}{K}\right)-\frac{g n p}{h+n} \\
& \frac{d p}{d t}=r_{2} p+\frac{e g n p}{h+n}-m p^{2}
\end{aligned}
$$

System (2) possesses four different equilibrium points: (i) the population free equilibrium $E_{0}=(0,0)$, (ii) the predator free equilibrium $E_{1}=(K, 0)$, (iii) the prey free equilibrium $E_{2}=\left(0, \frac{r_{2}}{m}\right)$, and (iv) the interior equilibrium $E_{*}\left(n_{*}, p_{*}\right)$ with $p_{*}=\frac{r_{1}}{g}\left(1-\frac{n_{*}}{K}\right)\left(h+n_{*}\right)$, and $n_{*}$ is a positive root of the equation

$$
n^{3}+a n^{2}+b n+c=0,
$$

where

$$
a=2 h-K, b=\frac{g K}{r_{1} m}+h^{2}-2 h K, c=h K\left(\frac{r_{2} g}{r_{1} m}-h\right) .
$$

It is clear that the equilibrium points $E_{0}, E_{1}$ and $E_{2}$ always exist. Let us denote

$$
\alpha=a^{2}-b \text { and } \beta=2 a^{2}-3 a b+c .
$$

Then the existence conditions of the interior equilibrium are obtained by using the criteria given by Murray (1989) as:

(i) If $\alpha>0$ and either $\beta=0$ or $|\beta| \leq 2 \alpha^{\frac{2}{3}}$, there is a possibility of the existence of zero, one, two or three non-trivial equilibria. It is to be mentioned here that this is a necessary but not sufficient condition to obtain three non-trivial equilibria. 
(ii) If $\alpha>0$ and $|\beta|>2 \alpha^{\frac{2}{3}}$ or $\alpha \leq 0$, we have at most one non-trivial equilibrium.

From the biological point of view (regarding pattern formation), the most interesting thing would be to study the stability of the interior equilibrium point $E_{*}$. The Jacobian matrix corresponding to $E_{*}$ can be written as:

$$
J=\left(\begin{array}{ll}
a_{11} & a_{12} \\
a_{21} & a_{22}
\end{array}\right),
$$

where $a_{11}=-\frac{r_{1} n_{*}}{K}+\frac{g n_{*} p_{*}}{\left(h+n_{*}\right)^{2}}, a_{12}=-\frac{g n_{*}}{h+n_{*}}, a_{21}=\frac{e g h p_{*}}{\left(h+n_{*}\right)^{2}}, a_{22}=-m p_{*}$.

The corresponding characteristic equation of $J$ is

$$
\lambda^{2}+A \lambda+B=0
$$

where

$$
\begin{gathered}
A=-\left(a_{11}+a_{22}\right)=\frac{r_{1} n_{*}}{K}+m p_{*}-\frac{g n_{*} p_{*}}{\left(h+n_{*}\right)^{2}}, \\
B=a_{11} a_{22}-a_{12} a_{21}=m p_{*}\left(\frac{r_{1} n_{*}}{K}-\frac{g n_{*} p_{*}}{\left(h+n_{*}\right)^{2}}\right)+\frac{e g^{2} h n_{*} p_{*}}{\left(h+n_{*}\right)^{3}} .
\end{gathered}
$$

Here $A$ and $B$ are the trace and determinant of $J$, respectively. Our main interest is to investigate the Turing instability of the system where the uniform steady state of the system without diffusion is stable, but it is unstable in the partial differential equations with diffusion terms. Now, the condition for the uniform steady state to be stable for the corresponding ordinary differential equation 2 is given by

$$
A>0 \text { and } B>0 \text {. }
$$

\subsection{Turing instability}

Here, the condition for Turing instability of the spatially positive steady state $E_{*}$ of system (1) will be investigated. Although, the Turing instability criterion is obtained following the standard analysis (Murray, 2003; Edelstein-Keshet, 1988; Okubo and Levin, 2001; Segel and Jackson, 1972), it is included here for the completeness of the text. To study this, let us consider the linearized form of system (1) about $E_{*}\left(n_{*}, p_{*}\right)$ as follows:

$$
\begin{aligned}
& \frac{\partial n_{1}}{\partial t}=a_{11} n_{1}+a_{12} p_{1}+D_{1}\left(\frac{\partial^{2} n_{1}}{\partial x^{2}}+\frac{\partial^{2} n_{1}}{\partial y^{2}}\right), \\
& \frac{\partial p_{1}}{\partial t}=a_{21} n_{1}+a_{22} p_{1}+D_{2}\left(\frac{\partial^{2} p_{1}}{\partial x^{2}}++\frac{\partial^{2} p_{1}}{\partial y^{2}}\right),
\end{aligned}
$$


where, $n=n_{*}+n_{1}, p=p_{*}+p_{1}$. Here, $\left(n_{1}, p_{1}\right)$ are small perturbations of $(n, p)$ about the interior equilibrium point $E_{*}\left(n_{*}, p_{*}\right)$. Now consider the solution of system (3) in the form

$$
\left(\begin{array}{l}
n_{1} \\
p_{1}
\end{array}\right)=\left(\begin{array}{c}
N_{k} \\
P_{k}
\end{array}\right) e^{\lambda_{1} t+i\left(\kappa_{x} x+\kappa_{y} y\right)}
$$

where $\lambda_{1}$ is the growth rate of perturbation in time $t, \kappa_{x}$ and $\kappa_{y}$ represent the wave numbers of the solution. The Jacobian matrix of the linearized system can be written as:

$$
\tilde{J}=\left(\begin{array}{cc}
a_{11}-D_{1}\left(\kappa_{x}^{2}+\kappa_{y}^{2}\right) & a_{12} \\
a_{21} & a_{22}-D_{2}\left(\kappa_{x}^{2}+\kappa_{y}^{2}\right)
\end{array}\right) .
$$

In the spatial model, the value of $\lambda_{1}$ depends on the sum of the square of wave numbers $\kappa_{x}^{2}+\kappa_{y}^{2}$ (Baurmann et al., 2004). As a result, both wave numbers affect the eigenvalues. This makes clear that some Fourier modes will vanish in the long-term limit whereas others will amplify. For the sake of simplicity, we can make use of $\lambda_{1}$ being rotational symmetric function on the $\left(\kappa_{x}, \kappa_{y}\right)$-plane and substitute $\kappa^{2}=\kappa_{x}^{2}+\kappa_{y}^{2}$ and obtain the results for the two-dimensional case from the one-dimensional formulation. Thus, the corresponding characteristic equation of system (1) is given by

$$
\lambda_{1}^{2}+\tilde{A} \lambda_{1}+\tilde{B}=0
$$

where

$$
\begin{aligned}
& \tilde{A}=A+\kappa^{2}\left(D_{1}+D_{2}\right), \\
& \tilde{B}=B-\left(a_{11} D_{2}+a_{22} D_{1}\right) \kappa^{2}+D_{1} D_{2} \kappa^{4} .
\end{aligned}
$$

Using the Routh-Hurwitz criterion, it appears that the equilibrium point $E_{*}$ is locally asymptotically stable in the presence of diffusion iff $\tilde{A}>0$ and $\tilde{B}>0$. Clearly, $A>0$ implies $\tilde{A}>0$. Therefore, diffusive instability occurs only in the case when $B>0$, but $\tilde{B}<0$. Hence, the condition for diffusive instability is given by

$$
H\left(\kappa^{2}\right)=D_{1} D_{2} \kappa^{4}-\left(a_{11} D_{2}+a_{22} D_{1}\right) \kappa^{2}+B<0 .
$$

This shows that diffusion can induce the loss of stability with respect to perturbations of certain wave numbers. Here, $H$ is a quadratic function of $\kappa^{2}$ and the graph of $H\left(\kappa^{2}\right)=0$ is a parabola. Let, the minimum of $H\left(\kappa^{2}\right)=0$ is reached at $\kappa^{2}=\kappa_{c}^{2}$, where $\kappa_{c}^{2}$ is given by 


$$
\kappa_{c}^{2}=\left(a_{11} D_{2}+a_{22} D_{1}\right) / 2 D_{1} D_{2} .
$$

Therefore, with the above value of $\kappa_{c}^{2}$, the condition for diffusive instability given in Eq. (5) can be written as

$$
\left(a_{11} D_{2}+a_{22} D_{1}\right)^{2}>4 D_{1} D_{2} B
$$

In explicit form, the condition becomes

$$
\left\{m p_{*} D_{1}+\left(\frac{r_{1} n_{*}}{K}-\frac{g n_{*} p_{*}}{\left(h+n_{*}\right)^{2}}\right) D_{2}\right\}^{2}>4 D_{1} D_{2}\left\{m p_{*}\left(\frac{r_{1} n_{*}}{K}-\frac{g n_{*} p_{*}}{\left(h+n_{*}\right)^{2}}\right)+\frac{e g^{2} h n_{*} p_{*}}{\left(h+n_{*}\right)^{3}}\right\}
$$

Since it is not prominent from analytic conditions how the local asymptotic stability and the Turing instability depend on $r_{2}$, further investigation in the form of numerical simulation is carried out in the following.

\subsection{Numerical simulation}

In this section, numerically it is examined how a generalist predator influences the system dynamics depending on the availability of the additional food source. Specifically, the growth rate of the predator due to the additional food, $r_{2}$, is varied and observe the changes in the dynamics of the system where the other parameter values are fixed at $r_{1}=2, K=10, g=2, h=5, e=0.25, m=0.016$. The bifurcation results are obtained by using the software XPPAUT and plotted in MATLAB, whereas the other figures are drawn by writing code in MATLAB.

First, the existence of equilibria (marked with filled black circles) and their stability are observed in the phase plane starting at $(n, p)=(2,4)$ (marked with open black circles) for different $r_{2}$. In Figure 1(a), n and p-nullclines, marked by the dashed blue and green lines, respectively, are plotted at $r_{2}=0$. There exist three different equilibria: (i) $E_{0}=(0,0), E_{1}=(10,0)$ (not shown in the figure) and $E^{*}=(1.05,5.41)$. Here, the eigenvalues of $E_{0}$ are 0 and 2 , and therefore it is unstable. The eigenvalues of $E_{1}$ are -2 and 0.33 , and therefore it is an unstable saddle. The eigenvalues of $E^{*}$ are $0.007 \pm 0.3456 i$, and therefore it is an unstable focus surrounded by a limit cycle. The trajectory approaching the limit cycle is shown by the red line. Figure 1(b) is drawn at $r_{2}=0.05$ having four different equilibria: (i) $E_{0}=(0,0), E_{1}=(10,0)$ (not shown in the figure), $E_{2}=(0,3.12)$ and $E^{*}=(0.35,5.16)$. Here, the eigenvalues of $E_{0}$ are 2 and 0.05 , and therefore it is an unstable node. The eigenvalues of $E_{1}$ are -2 and 0.3833 , and therefore it is an unstable saddle. The eigenvalues of $E_{2}$ are 0.75 and -0.05 , and 

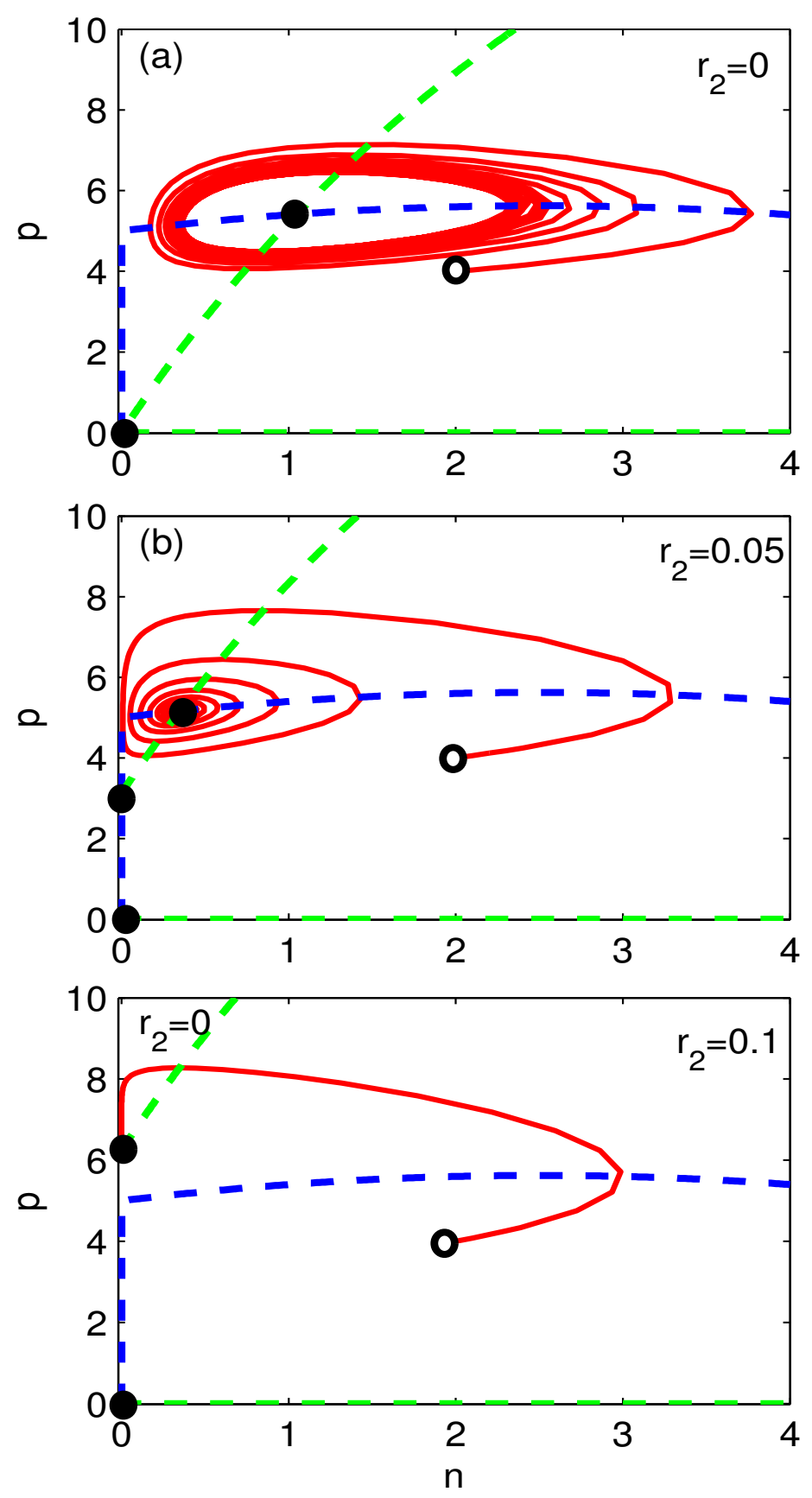

Figure 1: Phase plane of the model system 20 at different values of $r_{2}$ : (a) $r_{2}=0$, (b) $r_{2}=0.05$ and (c) $r_{2}=0.1$. Blue and green dashed lines are the $\mathrm{n}$ and p-nullclines, respectively. Different equilibria are marked by the filled black circles. Red lines are the corresponding trajectories starting at $(n, p)=(2,4)$, marked with open black circles. Parameter values used: $r_{1}=2, K=10, g=2, h=5, e=0.25, m=0.016$. (For interpretation of the references to colour in this figure legend, the reader is referred to the web version of this article.) 


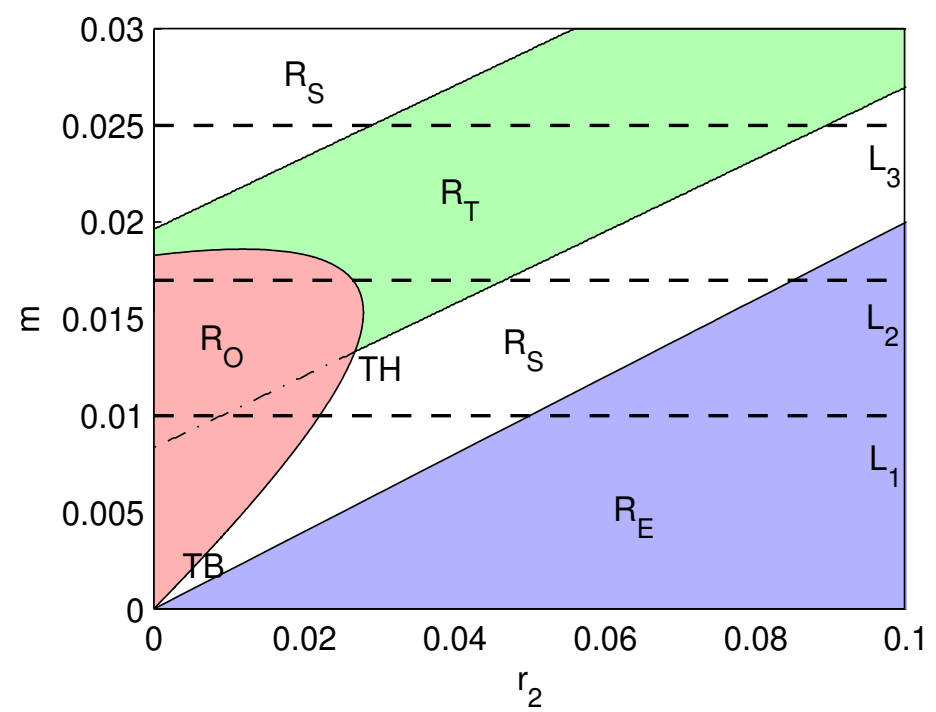

Figure 2: Two-dimensional bifurcation plot of model system (1) in $r_{2}-m$ plane which divides the $r_{2}-m$ parametric space into different regions; $R_{E}$ (blue): stable $E_{2} ; R_{S}$ (white): stable $E^{*} ; R_{O}$ (red): oscillating $E^{*}$; and $R_{T}$ (green): Turing instability. Here $T H$ and $T B$ are Turing-Hopf and Takens-Bogdanov bifurcations, respectively. The upper part of $R_{O}$ starting from the dashed-dot line is corresponding to the Turing-Hopf domain. Along the lines $L_{1}, L_{2}$ and $L_{3}$, Figs. 3 (a), -(c) are drawn, respectively. Parameter values used $D_{1}=0.01$ and $D_{2}=0.6$ and the other parameter values are same as in Fig. 1] (For interpretation of the references to colour in this figure legend, the reader is referred to the web version of this article.)

therefore it is also an unstable saddle. The eigenvalues of $E^{*}$ are $-0.0132 \pm 0.2324 i$, and therefore it is a stable focus and the corresponding trajectory reaching towards $E^{*}$ is shown by the red line. Next, Figure 1(c) is drawn at $r_{2}=0.1$. In this case, $E^{*}$ does not exist. The other equilibria are: (i) $E_{0}=(0,0), E_{1}=(10,0)$ (not shown in the figure), and $E_{2}=(0,6.25)$. The eigenvalues of $E_{0}$ are 2 and 0.1, and therefore it is an unstable node. The eigenvalues of $E_{1}$ are -2 and 0.43 , and therefore it is an unstable saddle. The eigenvalues of $E_{2}$ are -0.5 and -0.1 , and therefore it is a stable node and the corresponding trajectory reaching $E_{2}$ is shown by the red line.

To get a clearer view on how the presence of additional food source influences different dynamical behavior of the system, a two-parameter bifurcation diagram is drawn by varying the growth rate of the predator $\left(r_{2}\right)$ due to the additional food and the mortality of the predator $(m)$ (Figure 2). There are four different dynamical behaviors of the system marked by different regions $R_{E}, R_{S}, R_{O}$, and $R_{T}$. In region $R_{E}$ (marked by the blue color), $E_{2}$ is locally asymptotically stable (LAS), i.e., in this parametric region prey population becomes extinct due to high predation pressure and the predator 
population survives solely on the additional food source. Region $R_{S}$ (marked by the white color) is corresponding to the stable $E^{*}$, i.e., both the populations stably coexist in this parametric region. In region $R_{O}$ (marked by the red color), $E^{*}$ becomes unstable, and both the populations coexist with fluctuating densities. Region $R_{T}$ (marked by the green color) is the Turing space, i.e., in this region, $E^{*}$ remains stable for the system without diffusion, but becomes unstable in the presence of diffusion. As a result, different stationary spatially inhomogeneous patterns of predator and prey populations emerge within this region. The existence of two codimension-2 bifurcations are also observed, where the bifurcation curves interact. The first one is the Takens-Bogdanov bifurcation $(T B)$ where the Hopf bifurcation and transcritical bifurcation meet. The other one is Turing-Hopf bifurcation (TH) where the Turing bifurcation and Hopf bifurcation meet. The backward extended lower boundary of the Turing space, marked by the dash-dot line, divides the region $R_{O}$ into two parts. The upper part of this region is the Turing-Hopf domain where the inhomogeneous stationary patterns caused by the Turing instability interacts with the oscillations due to the Hopf bifurcation. Clearly, at lower rates of predator mortality, the presence of additional food to the predator helps in the stabilization of the system, whereas very high growth due to additional food results in prey extinction. On the other hand, when the mortality rate is comparatively high, the presence of additional food can make the distribution of the prey and predator inhomogeneous in space.

To get an overview of how prey abundance changes with $r_{2}$, three one-dimensional bifurcation diagrams are plotted (Figure 3 ) by varying $r_{2}$ continuously at (a) $m=0.01$, (b) $m=0.017$, and (c) $m=0.025$, which are drawn along the lines $L_{1}, L_{2}$ and $L_{3}$, respectively, as indicated in Figure 2 Specifically, the steady-state values of the abundances of the prey population are plotted with $r_{2}$. The black and red (dashed) lines indicate that the interior steady state is stable and unstable, respectively. The magenta (dashed) lines indicate that the steady state corresponding to the extinction of prey is stable. Additionally, the green lines represent the maximum and minimum abundances of the populations for the stable limit cycle. Color coding of the ranges of $r_{2}$ is same as in Figure2, From Figure 3 (a) it is clear that the prey population shows high fluctuation at low values of $r_{2}$. However, an increase in $r_{2}$ stabilizes system dynamics and finally prey population goes extinct from the system. In this case Turing instability does not occur. Figure 3 (b) shows a similar kind of behavior except for the 

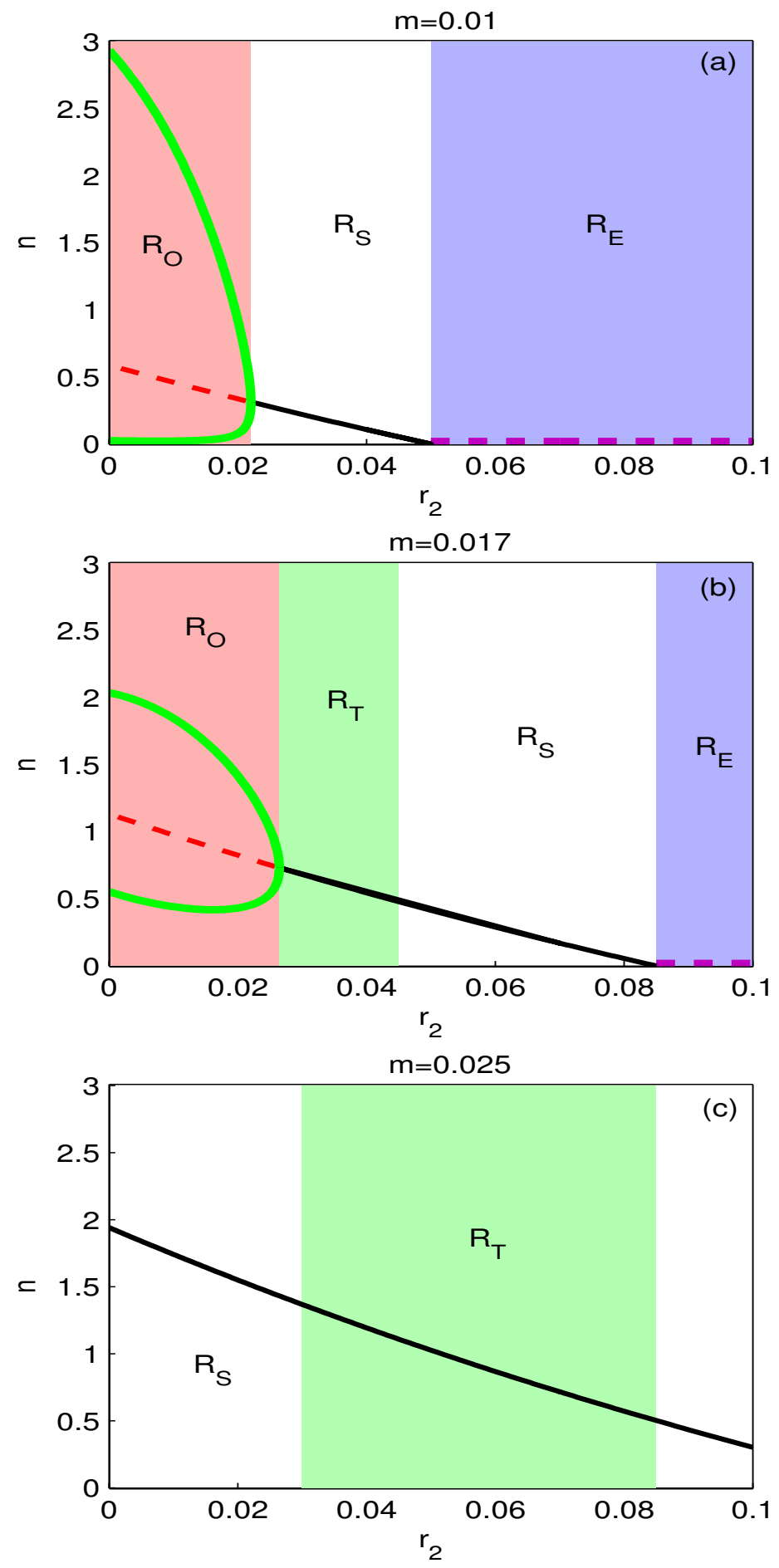

Figure 3: One-dimensional bifurcation diagrams to show how prey abundances change with $r_{2}$ at (a) $m=0.01$, (b) $m=0.017$ and (c) $m=0.025$. They are drawn along the lines $L_{1}, L_{2}$ and $L_{3}$, respectively, of Fig. 2, Color coding represents similar regions as that of Fig. 2. The black and red (dashed) lines indicate that $E^{*}$ is stable and unstable, respectively. The magenta (dashed) line is corresponding to stable $E_{2}$. The green lines represent the maximum and minimum abundances of the populations for the stable limit cycle. The other parameter values are same as in Fig. 2 (For interpretation of the references to colour in this figure legend, the reader is referred to the web version of this article.) 

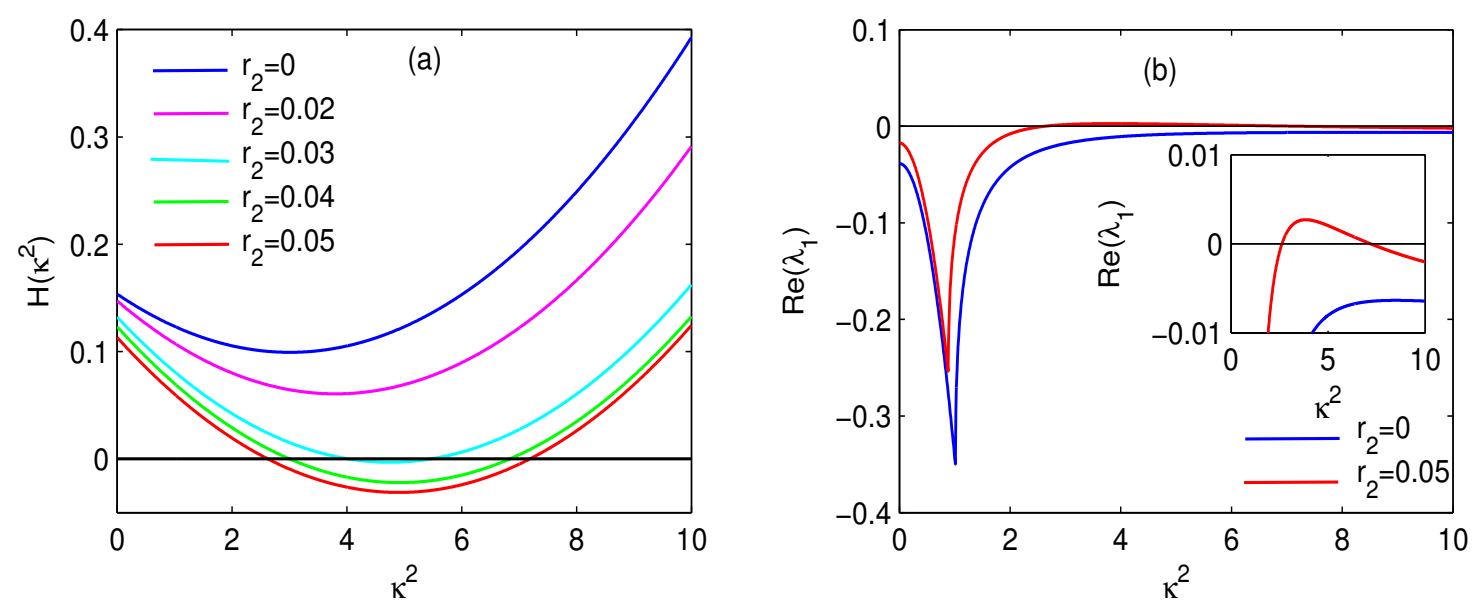

Figure 4: (a) The graph of the function $H\left(\kappa^{2}\right)$ at $r_{2}=0$ (blue), 0.02 (magenta), 0.03 (cyan), 0.04 (green) and 0.05 (red).

The other parameter values are same as in Fig. 3.c). An increase in the value of $r_{2}$ increases the possibility of diffusive instability by increasing the interval of negativity of $H\left(\kappa^{2}\right)$. (b) Dispersion relation plotting the largest real part of the eigenvalues at different $r_{2} ; r_{2}=0$ (blue) and $r_{2}=0.05$ (red). (For interpretation of the references to colour in this figure legend, the reader is referred to the web version of this article.)

range of $r_{2}$ just after the Hopf bifurcation where Turing instability occurs. For comparatively higher values of $m$, Figure 3(c) shows the non-existence of oscillating and prey-extinction regions. However, the range of $r_{2}$ for Turing instability is much larger compared to the previous case. In this case, the conditions of Turing instability obtained analytically (Eq. (5)) are also checked by plotting $H\left(\kappa^{2}\right)$ for different values of $r_{2}$. Turing instability condition $\min \left(H\left(\kappa^{2}\right)\right)<0$ is satisfied within the range $r_{2} \in(0.28,0.9)$. In Figure 4(a), the curve $H\left(k^{2}\right)=0$ is plotted for $r_{2}=0$ (blue), 0.02 (magenta), 0.03 (cyan), 0.04 (green) and 0.05 (red). The largest real parts of the eigenvalues of the characteristic equation (4) of system (1) are also drawn (Figure 4(b)) for $r_{2}=0$ (blue) and $r_{2}=0.05$ (red). The length of the interval of $\kappa^{2}$ within which the largest real part of the eigenvalues are positive provides the existence of diffusive instability.

In the following, different pattern formations are investigated at different values of $r_{2}$.

\subsection{Pattern formation}

Here, extensive numerical simulations of the spatial model system (1) are performed in two dimensional space using the forward finite difference method, and the results of different pattern formations due to the variation of $r_{2}$ are shown. 
To analyze the dynamic behavior of system (1), the stationary distributions of the prey population are plotted in two-dimensional spaces. Here, the system is studied on a squared spatial grid of $50 \times 50$ points with the Neumann boundary conditions and run the simulation up to the time $t=5000$ for different values of $r_{2}$. The space step is taken as 0.2 , and the time step as 0.005 . It is assumed that the prey and predator populations are spread over the whole domain at the beginning of the simulation. We know that the choice of the initial distribution of the populations greatly affects the spatial dynamics of a system. If the initial spatial distributions of the prey and predator are homogeneous, then the species distribution remains homogeneous forever, which is not so interesting (Petrovskii and Malchow, 1999). Apart from that, from a biological point of view, it is reasonable to consider a scattered nonuniform initial distribution of populations over the space under consideration. Here, such scattered initial distribution has been employed by considering a random sampling of the prey and predator populations around the equilibrium values of the corresponding non-spatial model. It is assured that the time at which simulations are stopped is sufficient for the patterns to attain the stationary state and they do not change further with time.

Figure 5 plots the stationary distribution of prey over the spatial domain for four different values of $r_{2}\left(r_{2}=0.032,0.037,0.045,0.08\right)$ keeping $m$ fixed at 0.025 . Specifically, $r_{2}$ is varied along the line $L_{3}$ in Figure 2 in such a way that $r_{2}$ lies within the Turing domain. It is to be mentioned here that, the distribution of the prey and predator remains homogeneous in space in the absence of additional food $\left(r_{2}=0\right)$ (the figure is not shown). Clearly, as $r_{2}$ increases, different types of dynamics emerge and it is observed that the distributions of prey and predator are always of the same type. Consequently, it is enough to show only the distributions of the prey for different $r_{2}$. At $r_{2}=0.032$, a cold spot pattern is observed. As we increase $r_{2}$, at $r_{2}=0.037$, the stripe pattern dominates the space. Again, at $r_{2}=0.045$, a mixture of hot spot and stripe patterns can be found, although hot spots dominate in this case. Finally, at $r_{2}=0.08$, we see stable hot spots with high prey densities in isolated zones.

\subsection{Spatiotemporal chaos}

Next, the spatial pattern formations of system (1) are examined by considering the parameters lying outside the Turing domain and inside the Hopf domain. Following the insightful work of Medvinsky et al. (2002), Wang et al. (2010), and Upadhyay et al. (2010), three different initial distributions are 

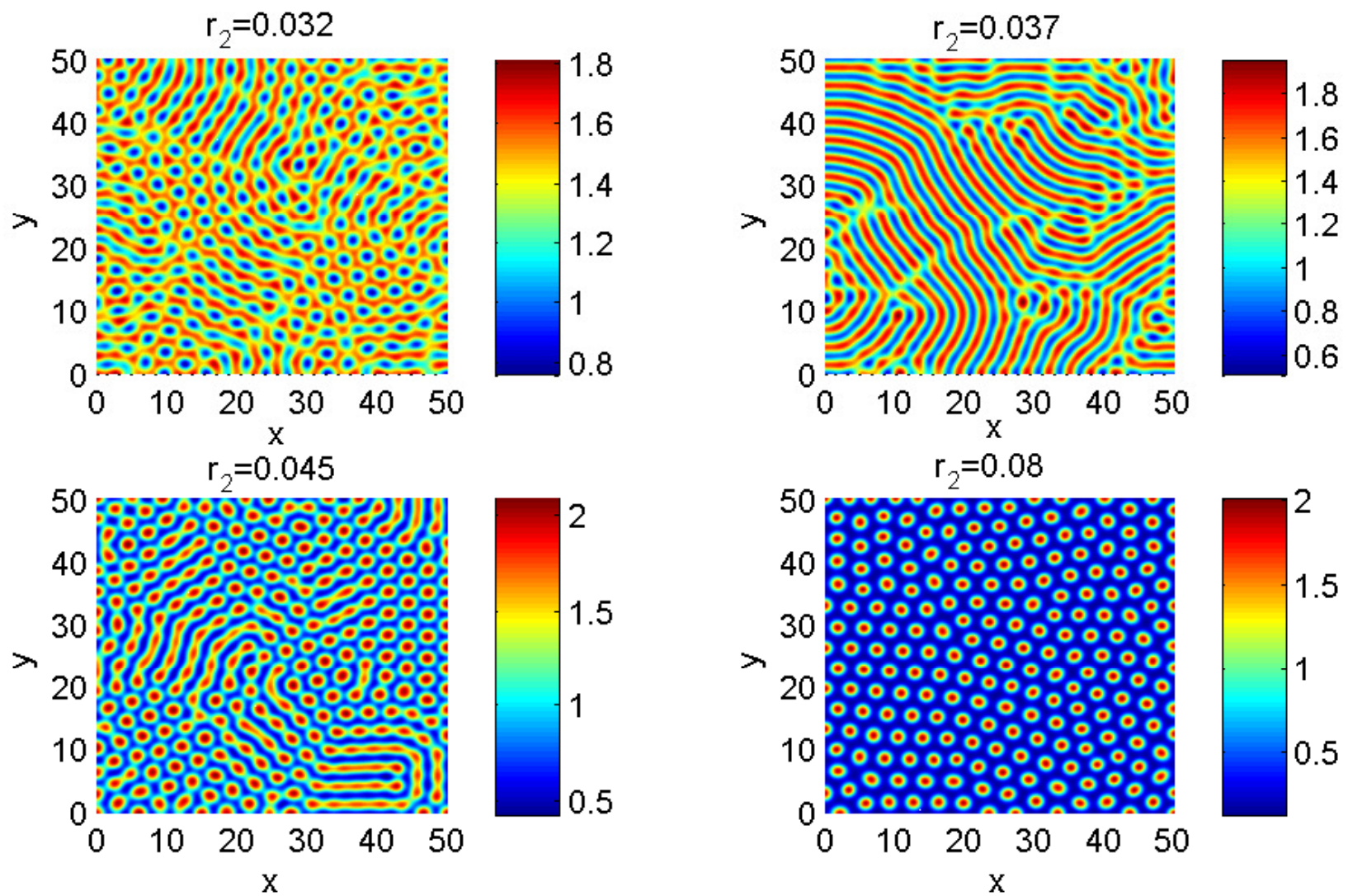

Figure 5: Stationary pattern formations of prey population over space at different values of $r_{2} ; r_{2}=0.032$ : cold spots; $r_{2}=0.037:$ stripes; $r_{2}=0.045:$ mixture of stripes and hot spots; $r_{2}=0.08:$ hot spots. Parameter values used $m=0.025, D_{1}=0.01, D_{2}=0.6$ and the other parameter values are same as in Fig. 2 


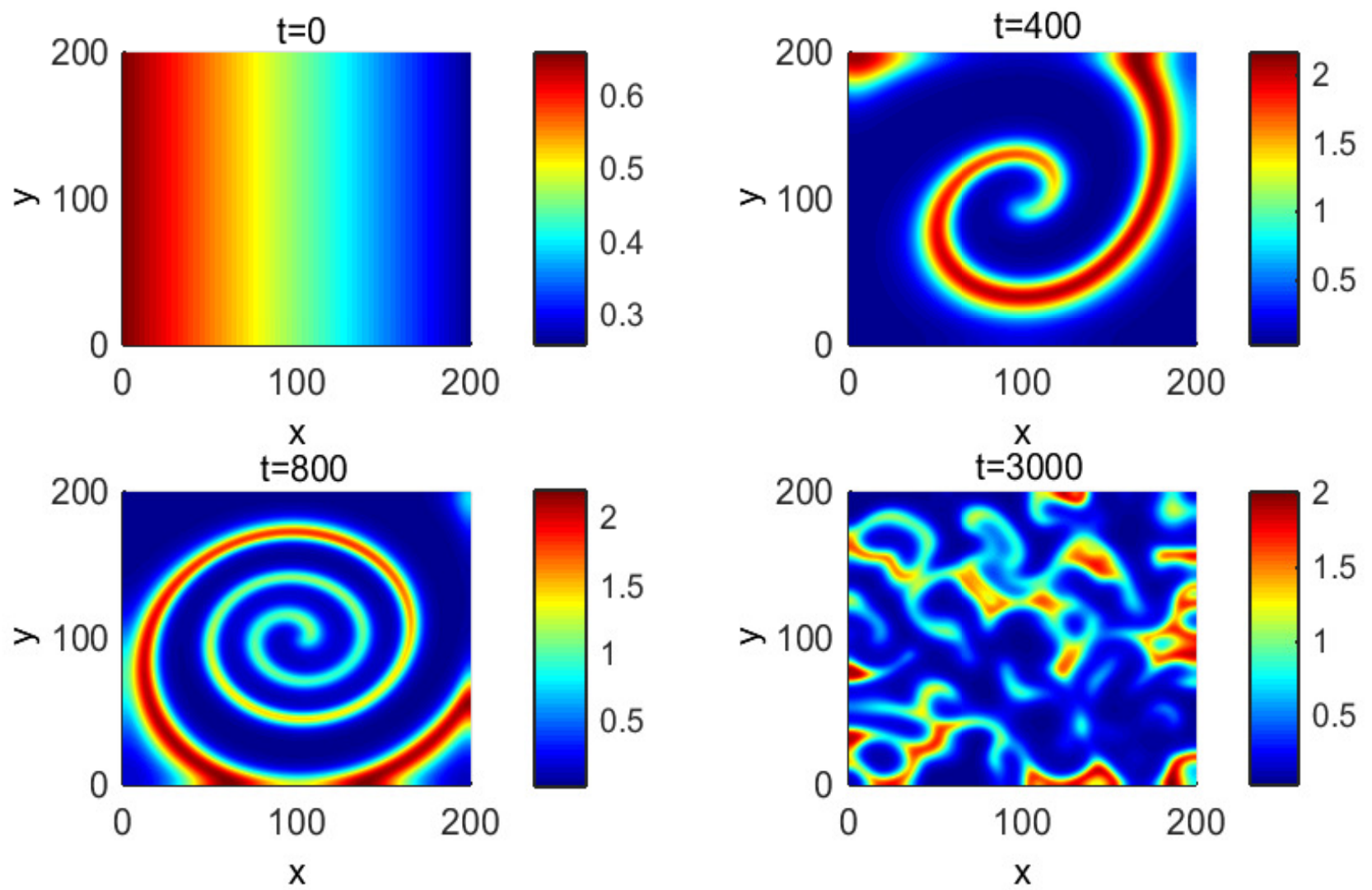

Figure 6: Formation of spiral pattern and its destruction for prey population at $t=0,400,800$, and 3000 . The parameter values used $m=0.01, r_{2}=0.01, D_{1}=0.1, D_{2}=0.2$ and the other parameters are same as in Fig. 2 and the initial distribution is given in Eq. (7).
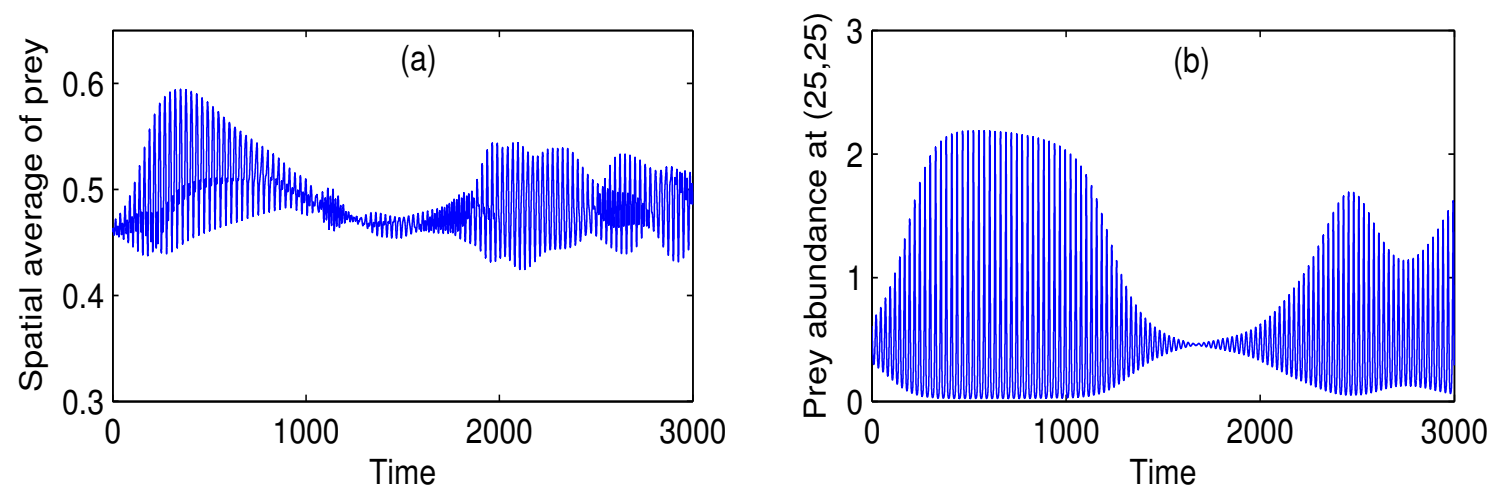

Figure 7: (a) Plot of spatial average values of prey population against time with parameter values same as in Fig. 6 showing chaotic oscillation. (b) Time evolution of prey population at the spatial location $(25,25)$. 
chosen to investigate the evolutionary process of the prey population in pattern formation. In this case, the system is studied on a squared spatial grid of $200 \times 200$ points and the parameters used are $r_{2}=0.01, m=0.01, D_{1}=0.1$, and $D_{2}=0.2$, whereas the other parameters are same as in Fig. 5 .

In the first case, the initial distribution of the populations is chosen as

$$
\begin{aligned}
& n(x, y, 0)=n^{*}-\varepsilon_{1}(x-100), \\
& p(x, y, 0)=p^{*}-\varepsilon_{2}(y-100),
\end{aligned}
$$

with $\varepsilon_{1}=2 \times 10^{-3}$ and $\varepsilon_{2}=3 \times 10^{-3}$. Snapshots of the spatial distributions are shown in Figure 6 for $t=0,400,800$, and 3000. Clearly, the formation of the irregular patchy structure can be preceded by the evolution of a regular spiral pattern. Here, the occurrence of the spiral is not due to the initial conditions. The center of the spiral is situated at the critical point $\left(x^{*}, y^{*}\right)=(100,100)$ with $n\left(x^{*}, y^{*}\right)=n^{*}, v\left(x^{*}, y^{*}\right)=p^{*}$. After the formation of the spiral, it grows upto a certain time, following the destruction of the spiral by making an irregular patchy pattern all over the domain.

Here, the distribution of the prey population does not converge to any stationary state. The spatial average of the prey population with time is plotted in Figure 7(a) which shows chaotic oscillation. The prey abundance at the spatial position $(25,25)$ is also plotted with respect to time in Figure 7 (b) which also shows an irregular oscillation with time.

In the second case, a different set of initial distribution of the populations is chosen as

$$
\begin{aligned}
& n(x, y, 0)=n^{*}-\varepsilon_{1}(x-40)(x-160)-\varepsilon_{2}(y-60)(y-140), \\
& p(x, y, 0)=p^{*}-\varepsilon_{3}(x-90)-\varepsilon_{4}(y-100),
\end{aligned}
$$

with $\varepsilon_{1}=3 \times 10^{-6}, \varepsilon_{2}=8 \times 10^{-6}, \varepsilon_{3}=3 \times 10^{-4}$, and $\varepsilon_{4}=6 \times 10^{-4}$. Snapshots of the spatial distribution are shown in Figure 8 for $t=0,600,900$, and 3000. Here, the initial distribution contains two critical points, which are $(40,140)$ and $(160,60)$. As a result, two spirals emerge with centers situated at the above mentioned points. In this case also the spiral pattern is destroyed and an irregular patchy pattern is formed all over the domain.

Finally, another set of initial distribution of the populations is considered as mentioned in the following

$$
n(x, y, 0)=n^{*}-\varepsilon_{1}(x-40)(x-160),
$$




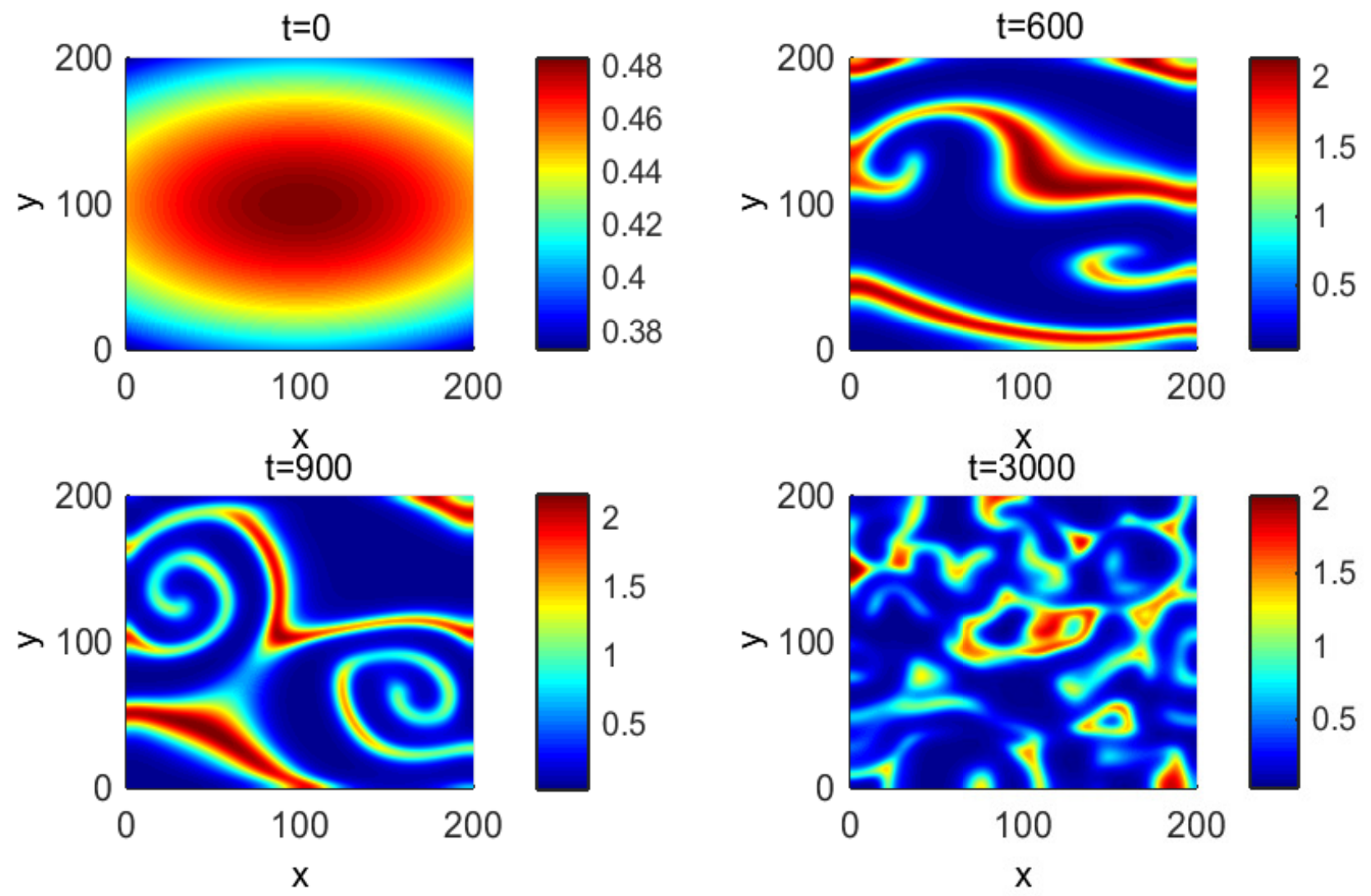

Figure 8: Formation of spiral pattern and its destruction for prey population at $t=0,600,900$, and 3000 with parameter values same as in Fig. 6 The initial distribution is given in Eq. 8. 

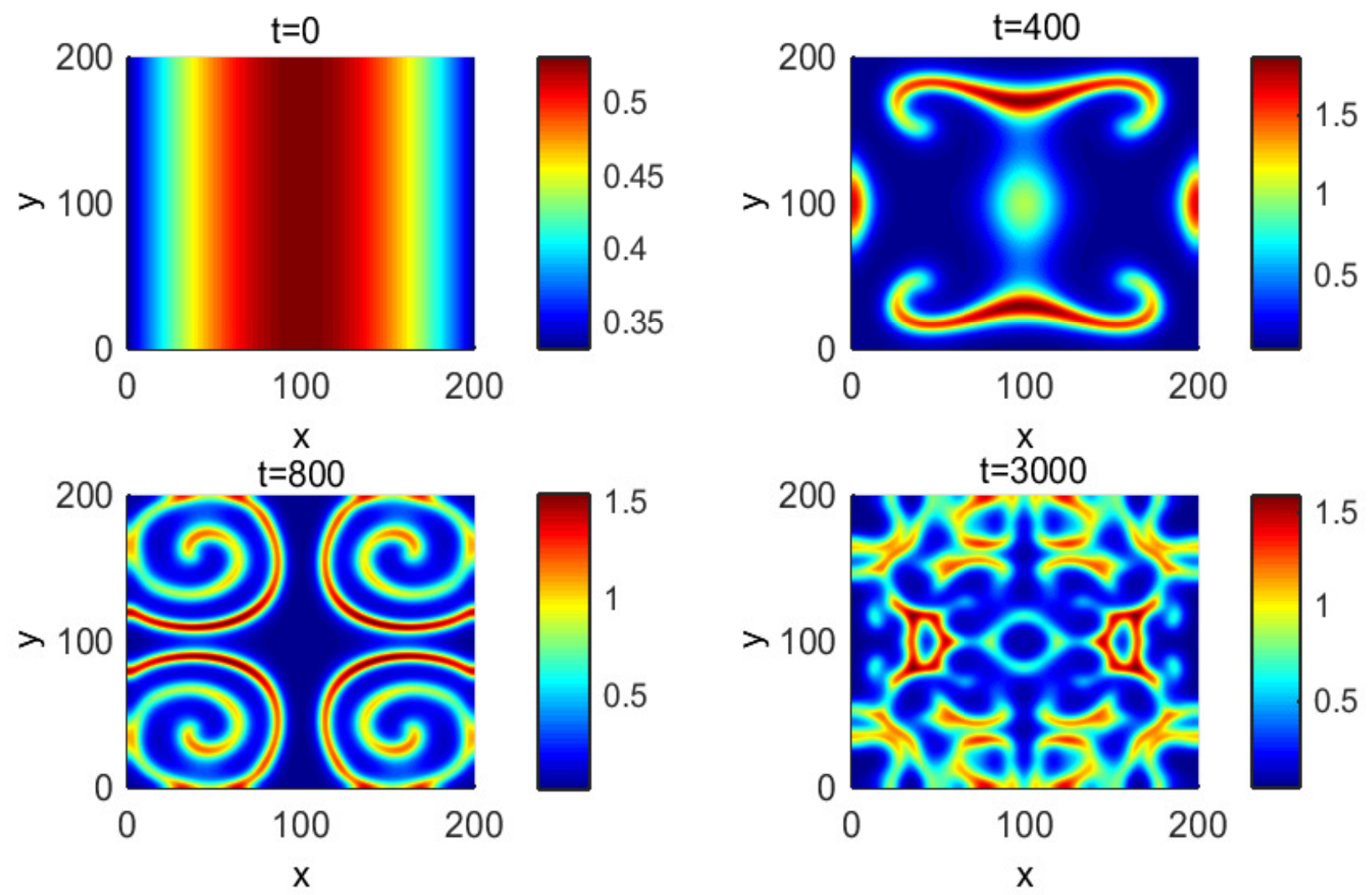

Figure 9: Formation of spiral pattern and its destruction for prey population at $t=0,400,800$, and 3000 with parameter values same as in Fig. 6 The initial distribution is given in Eq. 9. 


$$
p(x, y, 0)=p^{*}-\varepsilon_{2}(y-40)(y-160)
$$

with $\varepsilon_{1}=2 \times 10^{-5}$ and $\varepsilon_{2}=3 \times 10-5$. Snapshots of the spatial distribution are shown in Figure 9 for $\mathrm{t}=0,400,800$, and 3000. Here, the occurrence of four spirals is observed, which are finally destroyed and makes the spatial domain patchy.

\subsection{Density dependent birth rate for the generalist predator}

The behavior of system (1) is also checked by considering a density dependent birth rate of the generalist predator due to the additional food source (Erbach et al., 2013) in the form $\frac{r_{2} p}{h_{1}+p}$ where $h_{1}$ represents the half saturating constant for the growth of the predator due to the additional food source. In the absence of focal prey, the reproduction term of the predator population looks like Beverton-Holt function.

The behavior of the new system is checked at $h_{1}=1$. It is observed that the new system shows qualitatively similar spatial behavior as system (1). Only the difference is that the region of oscillation, $R_{O}$ (comparing with Figure 2 is relatively bigger and the prey extinction occurs at larger values of $r_{2}$.

\section{Model with intraguild predation}

In this section, a particular type of generalist predator is considered, called intraguild predator. In the case of intraguild predation, the additional food source of the predator coincides with the food source of the prey (Gagnon et al., 2011; Kang and Wedekin, 2013). System (1) can be modified in the presence of intraguild predation as:

$$
\begin{aligned}
& \frac{\partial n}{\partial t}=r_{1} n\left(1-\frac{n+\varepsilon p}{K}\right)-\frac{g n p}{h+n}+D_{1}\left(\frac{\partial^{2} n}{\partial x^{2}}+\frac{\partial^{2} n}{\partial y^{2}}\right) \\
& \frac{\partial p}{\partial t}=r_{2} \varepsilon p\left(1-\frac{n+\varepsilon p}{K}\right)+\frac{e g n p}{h+n}-m p^{2}+D_{2}\left(\frac{\partial^{2} p}{\partial x^{2}}+\frac{\partial^{2} p}{\partial y^{2}}\right)
\end{aligned}
$$

where $\varepsilon$ is the fraction of the predator population involved in intraguild predation. Clearly, $\varepsilon=0$ represents the situation where $p$ is not an intraguild (generalist) predator.

It is to be noted here that the intraguild predators share the same food as that of the prey population and as a result, they are involved in competition with the prey population for the common food source in addition to predate on them. A special kind of intraguild predation is known as mixotrophy where 
mixotrophs use a mix of different sources of energy and carbon, and because of that they compete with their prey organisms. Our mathematical form of intraguild predation is similar with the form used by Hammer and Pitchford (2005) where mixotrophy was explained in a phytoplankton-zooplankton system.

In the absence of diffusion, system (10) possesses four different equilibrium points: (i) the population free equilibrium $\bar{E}_{0}=(0,0)$, (ii) the predator free equilibrium $\bar{E}_{1}=(K, 0)$, (iii) the prey free equilibrium $\bar{E}_{2}=\left(0, \frac{r_{2} \varepsilon}{m}\right)$, and (iv) the interior equilibrium $\bar{E}_{*}\left(\bar{n}_{*}, \bar{p}_{*}\right)$ which can be obtained by solving the equations

$$
\begin{gathered}
r_{1}\left(1-\frac{n+\varepsilon p}{K}\right)-\frac{g p}{h+n}=0, \\
r_{2} \varepsilon\left(1-\frac{n+\varepsilon p}{K}\right)+\frac{e g n}{h+n}-m p=0 .
\end{gathered}
$$

Here, the condition for LAS of the non-diffusive version of system 100 is

$$
A_{1}>0 \text { and } B_{1}>0 \text {, }
$$

where

$$
\begin{aligned}
& A_{1}=-\left(a_{11}+a_{22}\right)=\frac{r_{1} \bar{n}_{*}}{K}+m \bar{p}_{*}-\frac{g \bar{n}_{*} \bar{p}_{*}}{\left(h+\bar{n}_{*}\right)^{2}}+\frac{r_{2} \varepsilon^{2} \bar{p}_{*}}{K}, \text { and } \\
& B_{1}=a_{11} a_{22}-a_{12} a_{21}=\left(m \bar{p}_{*}+\frac{r_{2} \varepsilon^{2} \bar{p}_{*}}{K}\right)\left(\frac{r_{1} \bar{n}_{*}}{K}-\frac{g \bar{n}_{*} \bar{p}_{*}}{\left(h+\bar{n}_{*}\right)^{2}}\right)+\left(\frac{r_{1} \varepsilon \bar{n}_{*}}{K}+\frac{g \bar{n}_{*}}{\left(h+\bar{n}_{*}\right)}\right)\left(-\frac{r_{2} \varepsilon \bar{p}_{*}}{K}+\frac{e g h \bar{p}_{*}}{\left(h+\bar{n}_{*}\right)^{2}}\right) .
\end{aligned}
$$

Here, $A_{1}$ and $B_{1}$ are the trace and determinant of the corresponding Jacobian, respectively. The condition for diffusive instability is given by

$$
H_{1}\left(\kappa^{2}\right)=D_{1} D_{2} \kappa^{4}-\left(\left(m \bar{p}_{*}+\frac{r_{2} \varepsilon^{2} \bar{p}_{*}}{K}\right) D_{1}+\left(\frac{r_{1} \bar{n}_{*}}{K}-\frac{g \bar{n}_{*} \bar{p}_{*}}{\left(h+\bar{n}_{*}\right)^{2}}\right) D_{2}\right) \kappa^{2}+B_{1}<0 .
$$

Following the same method as previous, it is possible to write down the explicit form of the condition for diffusive instability as

$$
\begin{aligned}
& \left\{\left(m \bar{p}_{*}+\frac{r_{2} \varepsilon^{2} \bar{p}_{*}}{K}\right) D_{1}+\left(\frac{r_{1} \bar{n}_{*}}{K}-\frac{g \bar{n}_{*} \bar{p}_{*}}{\left(h+\bar{n}_{*}\right)^{2}}\right) D_{2}\right\}^{2}> \\
& \quad 4 D_{1} D_{2}\left\{\left(m \bar{p}_{*}+\frac{r_{2} \varepsilon^{2} \bar{p}_{*}}{K}\right)\left(\frac{r_{1} \bar{n}_{*}}{K}-\frac{g \bar{n}_{*} \bar{p}_{*}}{\left(h+\bar{n}_{*}\right)^{2}}\right)+\left(\frac{r_{1} \varepsilon \bar{n}_{*}}{K}+\frac{g \bar{n}_{*}}{\left(h+\bar{n}_{*}\right)}\right)\left(-\frac{r_{2} \varepsilon \bar{p}_{*}}{K}+\frac{e g h \bar{p}_{*}}{\left(h+\bar{n}_{*}\right)^{2}}\right)\right\} .
\end{aligned}
$$

\footnotetext{
First, the condition of Turing instability obtained analytically in Eq. 111 is checked by plotting
} $H_{1}\left(\kappa^{2}\right)$ for different values of $\varepsilon$. In Figure 10 (top), the curve $H_{1}\left(k^{2}\right)=0$ is plotted for $\varepsilon=0$ (blue), 0.01 (magenta), 0.02 (cyan), 0.03 (green) and 0.04 (red). Clearly, the Turing instability condition 

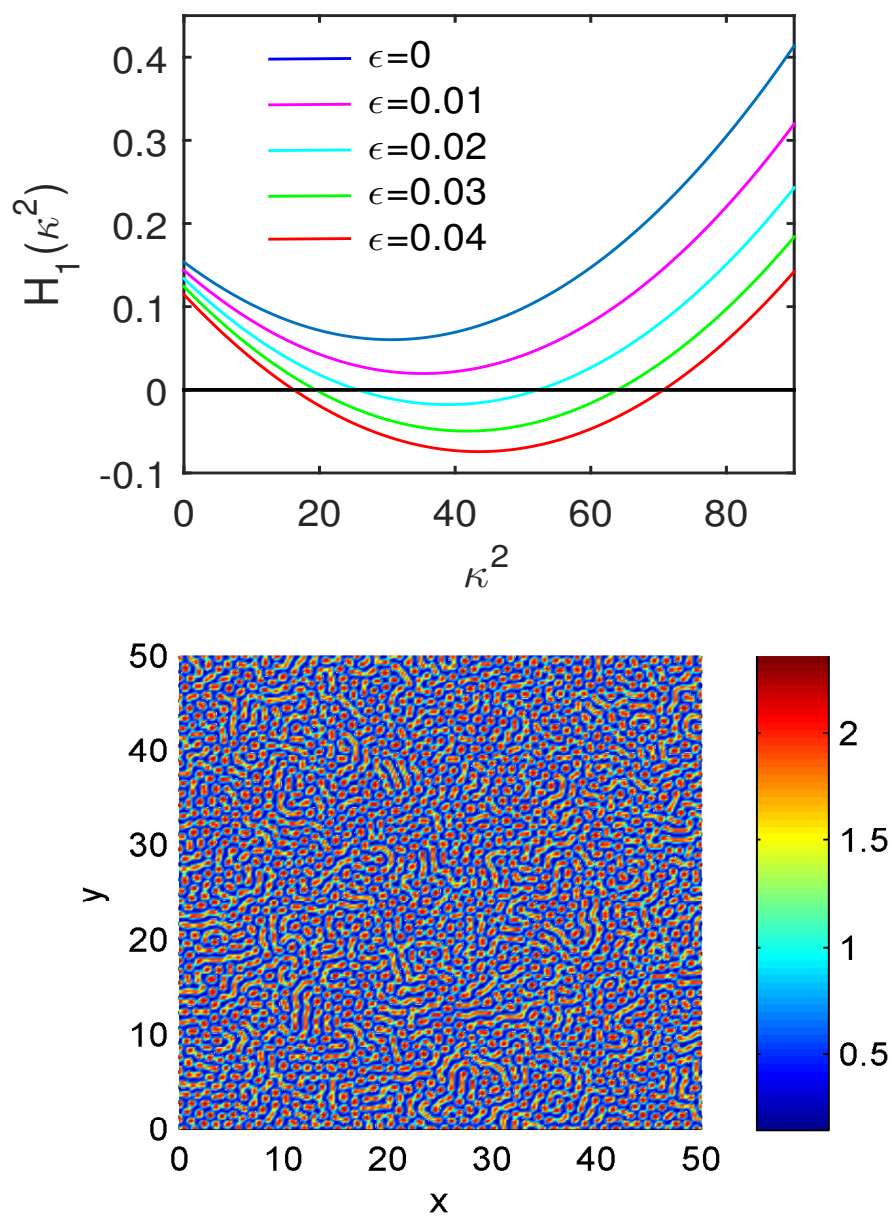

Figure 10: (Top) The graph of the function $H_{1}\left(\kappa^{2}\right)$ for system 10 at $\varepsilon=0$ (blue), 0.01 (magenta), 0.02 (cyan), 0.03 (green) and 0.04 (red). (Bottom) Stationary pattern formations of prey population over space at $\varepsilon=0.07$. The parameter values used $r_{1}=0.8, D_{1}=0.001, D_{2}=0.1$ and the other parameter values are same as in Fig. 4 (For interpretation of the references to colour in this figure legend, the reader is referred to the web version of this article.)

$\min \left(H_{1}\left(\kappa^{2}\right)\right)<0$ is satisfied for higher values of $\varepsilon$ which results in Turing pattern formation. Next, a numerical example of Turing pattern formation is shown. Figure 10 (bottom) is drawn at $\varepsilon=0.07$ which clearly shows stationary pattern formation by the prey population in the presence of intraguild predator.

\section{Discussion}

Predator-prey interactions affect species composition and community dynamics. The complexity in a community depends on the type of predation, which differs for different predators. Generalist predators increase such complexity by feeding on a variety of prey items. In the present work, the 
influences of two different types of generalist predators are investigated: (i) the predator is having an additional food source apart from the focal prey, and (ii) the predator is an intraguild predator where the additional food source coincides with the food of the prey, which results in a competition between the prey and the predator for the common food. Here, a separate growth term for the generalist predator is considered to represent its growth due to the additional food sources. The non-spatial version of the model shows stabilizing effect of generalist predators on system dynamics. However, the most interesting result occurs after considering diffusion in the model system in order to investigate the role of the generalist predator in the presence of spatial movements of both predator and prey populations. Although, the presence of the generalist predator assures temporal stability, the distribution of both prey and predator populations can become inhomogeneous in space and results in different patterns, like stripes, spots, and the mixture of them depending on the availability of the additional food to the generalist predator. Moreover, spatiotemporal chaotic patterns have also been observed for a certain range of the availability of additional food and mortality of the generalist predator.

Most of the previous modeling studies revealed the stabilizing role of generalist predators (Andersson and Erlinge, 1977; Turchin and Hanski, 1997; van Baalen et al., 2001; Smout et al., 2010). The presence of generalist predators results in the dampening or elimination of the cyclical interactions between predators and their prey (Hanski et al. 1991). Several empirical evidences also support this claim (Erlinge et al., 1983; Hanski et al., 1991). However, under certain conditions, it can also have destabilizing effects (Chakraborty and Chattopadhyay, 2008). Matthiopoulos et al. (2007) studied the interaction between a generalist predator Hen Harrier (Circus cyaneus) and three of its prey species in the United Kingdom, the Meadow Pipit (Anthus pratensis), the field vole (Microtus agrestis), and the Red Grouse (Lagopus lagopus scoticus). They found that the generalist predator can damp or suppress the cyclic oscillation in grouse population when the alternative prey density remains low. But, the presence of high alternative prey results in an increase in the oscillation. The present spatial system can also show a similar destabilizing effect on system dynamics in the presence of additional food. However, in this case, the destabilization occurs in space, whereas the temporal dynamics still remain stable. Under different conditions, additional food can also stabilize the system in both time 
and space.

The presence of generalist predators can make the system dynamics very complex. Previously, bistability between two alternative stable coexistence states, and bistability between a coexistence state and a stable limit cycle have been observed in a single prey-generalist predator system (Spencer and Collie, 1995). Magal et al. (2008) found the existence of homoclinic loops in the presence of generalist predator. Moreover, Erbach et al. (2013) found bistability, limit cycles and several global bifurcations in a simple predator-prey system with generalist predator. The present paper shows that, in spite of having less complex dynamics in the temporal model, the consideration of spatial inhomogeneity can result in different complex behaviors due to the presence of generalist predators. Addition of diffusion results in Turing instability, where the prey and predator populations oscillate in space although remain stationary in time. Similar kind of Turing instability was previously observed in a host-parasitoid model with generalist predation on host population by Wilson et al. (1999). They extended the Nicholson and Bailey model (1935) by incorporating the dispersal of both host and parasitoid offspring and found either stable pattern or rapid host extinction depending on the initial conditions. Generalist predation has also been observed to produce spatially varying stable patterns in the context of the McArthur-Resenzweig predator-prey model (Rosenzwig, 1973; Segel and Levin, 1976). However, the consideration of additional food source for the generalist predator which helps generalist predator to survive in the absence of focal prey makes the present approach more realistic and unique. Moreover, the existence of Turing-Hopf bifurcation and Takens-Bogdanov bifurcation is also observed, which are codimension-2 bifurcations resulting due to the interaction of Hopf and Turing bifurcations, and Hopf and transcritical bifurcations, respectively. The existence of spatiotemporal chaos in the presence of generalist predator is another interesting finding of the present work. Previously, Kumari (2013) observed the existence of chaos in a spatial prey-predator-top predator system where the top predator was considered as the generalist predator. In the present case, chaos occurs in a parametric range that falls outside the Turing domain. Such generation of chaotic patterns outside the Turing domain was found in some of the previous studies without generalist predators (Baurmann et al., 2007; Banerjee and Petrovskii, 2011; Banerjee and Abbas, 2014).

Spatial variations in population densities due to the variation of extrinsic factors such as nutrient 
concentration, moisture and temperature, are normal phenomena in ecological systems. In comparison, empirical evidences of intrinsically generated fixed spatial patterns are difficult to identify as it is hard to neglect the extrinsic factors as well as the difficulty in accurately estimating the key interactions and dispersal parameters. In spite of such difficulty, researchers found several evidences of spatial pattern formations due to biological factors. For example, the clustered spatial pattern of ant nests emerges from the natural history of the ant/scale/beetle interaction (Liere et al. 2012). With the help of experimental and modeling studies, Shiyomi (1980) showed that the spatial pattern of a population of Galleria mellonella is affected by the frequency of attack by the predator Podisus maculiventris (attack ability), the homogeneity of the attack ability within a predator population and the mobility of the predator. There are also evidences of spatial pattern formation due to the predation by generalist predators. In a field study, Winder et al. (2005) found a deep impact of spatial distribution of cereal aphids in the presence of two generalist predators, Pterostichus melanarius and P. madidus. These observations support the findings of the present study regarding the possibility of pattern formation in the presence of generalist predators.

Generalist predators have important ecological impacts and wide applicability in the field of biological control. In practice, generalist predators are used to control the populations of ecologically damaging species, particularly of agricultural weed and insect pests (DeBach, 1974; Holt and Hochberg, 1997). Such biological controls are environment friendly alternatives for the use of insecticides. However, the success in controlling damaging species depends on the preferences of the generalist predator for the focal prey and alternative food (Koss and Snyder, 2005) as well as on the spatial and temporal scales at which the process is studied (Walde, 1994). In this respect, theoretical studies can provide significant insights in finding optimal strategies for control mechanisms. Previously, Magal et al. (2008) examined conditions under which the invasion of leafminers can be stopped and reversed by generalist parasitoid in spatial scale. The present study reveals that the theoretical prediction of a temporal model can go horribly wrong in real systems where populations are involved in spatial movements. In the presence of a generalist predator, the system can show different pattern formations and spatiotemporal chaos which has important implications for ecosystem functioning not only in terms of their predictability, but also in influencing species persistence (Huisman and Weissing, 1999) 


7

and ecosystem's stability in response to abrupt environmental changes (Petrovskii et al., 2004). The relevance of investigating the role of generalist predators in spatially extended domain was recently mentioned by Erbach et al. (2013).

To the best of our knowledge, the present paper is the first possible theoretical work showing different pattern formations due to the presence of generalist predators. In nature, predator-prey systems are more complex than what a simple two dimensional model can capture. Further investigation and empirical support are needed to confirm the importance of generalist predators in spatial scale. Our next step would be to investigate the effects of generalist predators in the presence of a specialist predator. In that case, the generalist predator would be either sharing food with the focal prey or simply depend on the additional food different from the food source of the focal prey in addition to compete with the specialist prey for the focal prey. To determine proper optimal strategy for biological control we need to examine different mechanisms of pattern formation as they mimic the processes of ecological patterning in real world ecosystems.

Acknowledgment: The author wishes to thank the Alexander von Humboldt Foundation for financial support in the form of a postdoctoral fellowship at the ICBM, Carl von Ossietzky University, Oldenburg, Germany. The author also received support from HCØ postdoctoral fellowship program for a part of this work. The author is grateful to the reviewers for their insightful comments and suggestions on the previous versions of the manuscript.

[1] Andersson, M., S. Erlinge., 1977. Influence of predation on rodent populations. Oikos 29, 591-597.

[2] Banerjee, M., Abbas, S., 2014. Existence and non-existence of spatial patterns in a ratiodependent predator-prey model. Ecological Complexity. doi:10.1016/j.ecocom.2014.05.005

[3] Banerjee, M., Petrovskii, S., 2011. Self-organised spatial patterns and chaos in a ratio-dependent predator-prey system. Theor. Ecol. 4, 37-53.

[4] Baurmann, M., Ebenhoh, W., Feudel, U., 2004. Turing instabilities and pattern formation in a benthic nutrient-microorganism system. Math. Biosci. Eng. 1, 111-130.

[5] Baurmann, M., Gross, T., Feudel, U., 2007. Instabilities in spatially extended predator-prey 
systems: Spatio-temporal patterns in the neighborhood of Turing-Hopf bifurcations. J. Theor. Biol. 245, 220-229.

[6] Chakraborty, S., Chattopadhyay, J., 2008. Nutrient-phytoplankton-zooplankton dynamics in the presence of additional food sourceA mathematical study. Journal of Biological Systems 16 (04), $547-564$.

[7] Chakraborty, S., Feudel, U., 2014. Harmful algal blooms: combining excitability and competition. Theoretical Ecology 7 (3), 221-237.

[8] Chakraborty, S., Tiwary, P., Misra, A., Chattopadhyay, J., 2015. Spatial dynamics of a nutrientphytoplankton system with toxic effect on phytoplankton. Mathematical Biosciences 264, 94-100.

[9] Crowder, D.W., Snyder, W.E., 2010. Eating their way to the top? Mechanisms underlying the success of invasive generalist predators. Biol. Invas. 12, 2857-2876.

[10] DeBach, P., 1974. Biological Control by Natural Enemies, Cambridge University Press, UK.

[11] Edelstein-Keshet, L., 1988. Mathematical Models in Biology, Birkhauser Mathematical Series.

[12] Erbach, A., Lutscher, F., Seo. G., 2013. Bistability and limit cycles in generalist predator-prey dynamics. Ecological Complexity 14, 48-55.

[13] Erlinge, S., Gransson, G., Hansson, L., 1983. Predation as regulating factor on small rodent populations in southernmost Sweden. Oikos 40, 36-52.

[14] Gagnon, AÉ., Heimpel, GE., Brodeur, J., 2011. The Ubiquity of Intraguild Predation among Predatory Arthropods. PLoS ONE 6(11), e28061.

[15] Hammer, A.C., Pitchford, J.W., 2005. The role of mixotrophy in plankton bloom dynamics, and the consequences for productivity. ICES Journal of Marine Science 62, 833-840.

[16] Hanski, I., Hansson, L., Henttonen, H., 1991. Specialist predators, generalist predators, and the microtine rodent cycle. Journal of Animal Ecology 60, 353-367.

[17] Hesaaraki, M., Moghadas, S.M., 2001. Existence of limit cycles for predator-prey systems with a class of functional responses. Ecological Modelling 142, 1-9. 
[18] Holt, R.D., Hochberg, M., 1997. When is biological control evolutionarily stable (or is it)? Ecology $78,1673-1683$.

[19] Huisman, J., Weissing, F.J., 1999. Biodiversity of plankton by oscillations and chaos. Nature 402, 407-410

[20] Jaworski, CC., Bompard, A., Genies, L., Amiens-Desneux, E., Desneux, N., 2013. Preference and Prey Switching in a Generalist Predator Attacking Local and Invasive Alien Pests. PLoS ONE 8(12), e82231.

[21] Kang Y., Wedekin L., 2013. Dynamics of a intraguild predation model with generalist or specialist predator. J. Math. Biol. 67, 1227-1259.

[22] Kar, T.K., Matsuda, H., 2007. Global dynamics and controllability of a harvested prey-predator system with Holling type III functional response. Nonlinear Analysis: Hybrid Systems 1, 59-62.

[23] Koss, A.M., Synder, W.E., 2005. Alternative prey disrupt biocontrol by a guild of generalist predators. Biol. Control. 32, 243-251.

[24] Kumari, N., 2013. Pattern Formation in Spatially Extended Tritrophic Food Chain Model Systems: Generalist versus Specialist Top Predator. ISRN Biomathematics, Article ID 198185, 12 pages

[25] Liere, H., Jackson, D., Vandermeer, J., 2012. Ecological Complexity in a Coffee Agroecosystem: Spatial Heterogeneity, Population Persistence and Biological Control. PLoS ONE 7(9), e45508.

[26] Magal, C., Cosner, C., Ruan, S., Casas, J., 2008. Control of invasive hosts by generalist parasitoids. Mathematical Medicine and Biology 25, 1-20.

[27] Malchow, H., Petrovskii, S., Venturino, E., 2008. Spatiotemporal patterns in Ecology and Epidemiology: Theory, Models, Simulations. Chapman \& Hall / CRC Press.

[28] Matthiopoulos, J., Graham, K., Smout, S., Asseburg, C., Redpath, S., Thirgood, S., Hudson, P. and Harwood, J., 2007. Sensitivity to assumptions in models of generalist predation on a cyclic prey. Ecology 88, 2576-2586. 
[29] Medvinsky, A.B., Petrovskii, S.V., Tikhonova, I.A., Malchow, H., Li, B.L., 2002. Spatiotemporal complexity of plankton and fish dynamics. SIAM Rev. 44, 311-370.

[30] Morozov, A., Petrovskii, S., 2009. Excitable population dynamics, biological control failure, and spatiotemporal pattern formation in a model ecosystem. Bulletin of Mathematical Biology 71, 863-887.

[31] Murray, J.D., 1989. Mathematical Biology. New York: Springer.

[32] Murray, J.D., 2003. Mathematical Biology, Springer-Verlag, 3rd edition.

[33] Nicholson, AJ., and Bailey, VA., 1935. The balance of animal population. Proceedings of the zoological society of London 1, 551-598.

[34] Okubo, A., and Levin, S.A., 2001. Diffusion and Ecological Problems: modern Perspectives (2nd edition). New York, Springer-Verlag.

[35] Petrovskii, S.V., Li, B.L., Malchow, H., 2004. Transition to spatiotemporal chaos can resolve the paradox of enrichment. Ecol. Complexity. 1, 37-47.

[36] Petrovskii, S.V., Malchow, H., 1999. A minimal model for pattern formation in a prey-predator system. Math. Comput. Model. 29, 49-63.

[37] Polis, G., McCormick, S. 1987. Intraguild predation and competition among desert scorpions. Ecology 68, 332-343.

[38] Polis, GA., Meyers, CA., Holt, RD., 1989. The ecology and evolution of intraguild predation: potential competitors that eat each other. Annual Review of Ecology and Systematics 20, 297330.

[39] Rosenheim, J.A., Kaya, H.K., Ehler, L.E., Marois, J.J., Jaffee, B.A., 1995. Intraguild predation among biological control agents: theory and evidence. Biol. Control 5, 303-335.

[40] Rosenzweig, M.L., 1971. Paradox of enrichment: destabilization of exploitation ecosystems in ecological time. Science 171, 385-387.

[41] Rosenzweig, M.L., 1973. Evolution of the predator isocline. Evolution 27, 84-94. 
[42] Segel, L.A., and Jackson, J.L., 1972. Dissipative structure: An explanation and an ecological example. J. Theor. Biol. 37, 545-559.

[43] Segel, L.A., Levin, SA., 1976. Application of nonlinear stability theory to the study of the effects of diffusion on predator-prey interactions. Selected topics in statistical mechanics and biophysics (ed. R. Piccirelli) Conference Proceedings No. 27. AIP, New York.

[44] Seuront, L., 2009. Fractals and multifractals in ecology and aquatic science. Chapman \& Hall, London.

[45] Shiyom, M., 1980. Predation-affected spatial pattern changes in a prey population. Ecol. Modelling. 11, 1-14.

[46] Smout, S., Asseburg, C., Matthiopoulos, J., et al., 2010. The Functional Response of a Generalist Predator. PLoS ONE 5(5), e10761. doi:10.1371/journal.pone.0010761

[47] Spencer, P.D., Collie, J.S., 1995. A simple predator-prey model of exploited marine fish populations incorporating alternative prey. ICES Journal of Marine Science 53, 615-628.

[48] Steele, J.H., and Henderson, E.W., 1981. A simple plankton model. Am. Nat. 117, 676-691.

[49] Steele, J.H., Henderson, E.W., 1992. The role of predation in plankton models. Journal of Plankton Research 14, 157-172.

[50] Symondson, W.O.C., Sunderland, K.D., Greenstone, M.H., 2002. Can generalist predators be effective biocontrol agents? Annu. Rev. Entomol. 47, 561-594.

[51] Turchin, P., Hanski, I., 1997. An empirically based model for latitudinal gradient in vole population dynamics. American Naturalist 149, 842-874.

[52] Upadhyay, R.K., Wang, W., Thakur, N.K., 2010. Spatiotemporal dynamics in a spatial Plankton system. Math. Modelling Nature Phenom 5, 102-122.

[53] van Baalen, M., Křivan, V., van Rijn, P.C.J., Sabelis, M.W., 2001. Alternative Food, Switching Predators, and the Persistence of Predator-Prey Systems. American Naturalist 157, 512-524. 
[54] van Leeuwen, E., Jansen, V.A.A., Bright, P.W., 2007. How population dynamics shape the functional response in a one-predator-two-prey system. Ecology 88, 1571-1581.

[55] Walde, S., 1994. Immigration and the dynamics of a predator-prey interaction in biological control. J. Anim. Ecol. 63, 337-346.

[56] Wang, W., Zhang, L., Wang, H., Li, Z., 2010. Pattern formation of a predator-prey system with Ivlev-type functional response. Ecol. Modelling 221, 131-140.

[57] Wilson, WG., Harrison, SP., Hastings, A., McCann, K., 1999. Exploring stable pattern formation in models of tussock moth populations. J. Anim. Ecol. 68, 94-107.

[58] Winder, L., Alexander, CJ., Holland, JM. Symondson, WOC., Perry, JN., Woolley, C., 2005. Predatory activity and spatial pattern: the response of generalist carabids to their aphid prey. Journal of Animal Ecology 74, 443-454.

[59] Xu, R., Chen, L., Chaplain, M., 2004. Persistence and global stability in a delayed predator-prey system with Holling-type functional response. ANZIAM Journal 46, 121-141. 